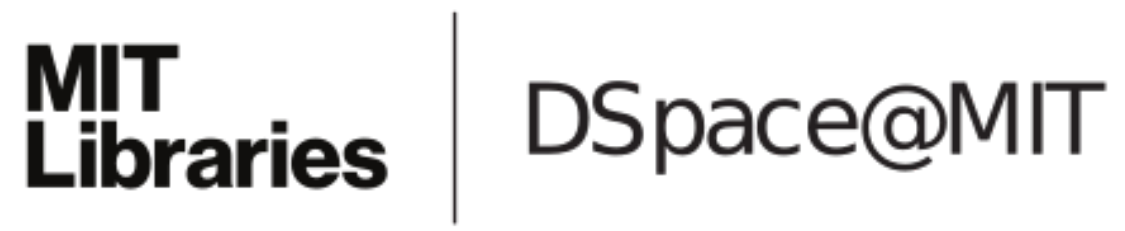

MIT Open Access Articles

Inkjet-printed conductive patterns for physical manipulation of audio signals

The MIT Faculty has made this article openly available. Please share how this access benefits you. Your story matters.

Citation: Gong, Nan-Wei, Amit Zoran, and Joseph A. Paradiso. "Inkjet-Printed Conductive Patterns for Physical Manipulation of Audio Signals." Proceedings of the Adjunct Publication of the 26th Annual ACM Symposium on User Interface Software and Technology - UIST '13 Adjunct (2013), October 8-11, 2013, St. Andrews, UK.

As Published: http://dx.doi.org/10.1145/2508468.2514932

Publisher: Association for Computing Machinery

Persistent URL: http://hdl.handle.net/1721.1/92457

Version: Author's final manuscript: final author's manuscript post peer review, without publisher's formatting or copy editing

Terms of use: Creative Commons Attribution-Noncommercial-Share Alike 


\section{Inkjet-printed Conductive Patterns for Physical Manipulation of Audio Signals}

\author{
Nan-Wei Gong \\ MIT Media Lab \\ Cambridge, MA, USA \\ nanwei@media.mit.edu
}

\author{
Amit Zoran \\ MIT Media Lab \\ Cambridge, MA, USA \\ amitz@media.mit.edu
}

\author{
Joseph A. Paradiso \\ MIT Media Lab \\ Cambridge, MA, USA \\ joep@media.mit.edu
}

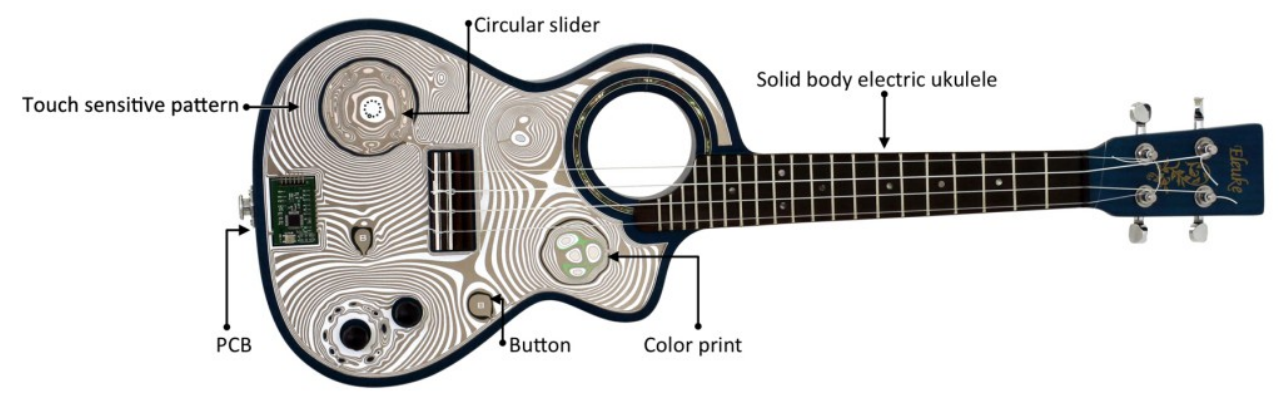

Figure 1 An example of inkjet-printed conductive patterns for physical manipulation of electric ukulele signals

\begin{abstract}
In this demo paper, we present the realization of a completely aesthetically driven conductive image as a multi-modal music controller. Combining two emerging technologies - rapid prototyping with an off-the-shelf inkjet printer using conductive ink and parametric graphic design, we are able to create an interactive surface that is thin, flat, and flexible. This sensate surface can be conformally wrapped around a simple curved surface, and unlike touch screens, can accommodate complex structures and shapes such as holes on a surface. We present the design and manufacturing flow and discuss the technology behind this multi-modal sensing design. Our work seeks to offer a new dimension of designing sonic interaction with graphic tools, playing and learning music from a visual perspective and performing with expressive physical manipulation.
\end{abstract}

\section{Author Keywords}

Sensate surface, music controller, customizable controller surface, flexible printed electronics, electronic skin

\section{ACM Classification Keywords}

H.5.2 [Information interfaces and presentation]: User Interfaces

\section{INTRODUCTION}

Traditional music instruments are beautiful objects. Great effort is often put into both their aesthetic presentation as

Permission to make digital or hard copies of all or part of this work for personal or classroom use is granted without fee provided that copies are not made or distributed for profit or commercial advantage and that copies bear this notice and the full citation on the first page. Copyrights for components of this work owned by others than ACM must be honored. Abstracting with credit is permitted. To copy otherwise, or republish, to post on servers or to redistribute to lists, requires prior specific permission and/or a fee. Request permissions from permissions@acm.org.

UIST'13 Adjunct, October 8-11, 2013, St. Andrews, United Kingdom.

Copyright (C) 2013 ACM 978-1-4503-2406-9/13/10 ..\$15.00.

DOI string from ACM form confirmation. well as sound quality. Modern sensing technology, however, can enable the instrument's decoration to also serve as an expressive controller. For example, circular patterns can be rotary controllers, stripes can serve as multitouch triggers, solid shapes can sense non-contact gesture \& pressure, etc. What was only for beauty now becomes sensate.

To explore the intersection between visual and auditory understanding and performance, we developed a music controller surface that covers an instrument with parametrically designed conductive patterns. The patterns function both as decoration and control inputs. And a player learns through physically sliding, tapping, and pressing into the surface, or bridging patterns for more dynamic effects.

Our work started with the experimentation of conductive inkjet-printed patterns as sensor-input controller surfaces with off-the-shelf inkjet printers. By combining color pigment and silver nanoparticles in inkjet printing, we are able to create an aesthetically-driven sensing surface that is part of the music instrument itself, while also functioning as a controller. Adapting parametric design, the sensing patterns are generated computationally.

We demonstrate the creation of a controller surface for an electric ukulele. One can learn to play it over time, as a user associates hand movements in different places with the different kind of effects and sounds. Each custom pattern allows players to "see" what they "hear" as they sculpt their timbre by touching \& gliding, mix signals through touches with multiple fingers, and physically remap the properties of sonic interaction in the computer software. 


\section{RELATED WORK}

Our motivation is driven by the exploration of the intimate intersection of aesthetic visual design with sound control and audio performance. An early example is The Optophonic Piano. Created by Vladimir Rossiné [1], it spun an aesthetically-driven painting, generating sound via an embedded light sensor as the picture was scanned. Gong et. al [2] started our exploration with conductive inkjet printing and the graphic design of flexible electronics as sensing input devices for various applications such as DIY music controllers. In a recent example, Freed \& Rowland made an aesthetic multi-electrode conductive pattern into a distributed speaker "browsed" with a handheld magnet [3].

\section{SYSTEM OVERVIEW}

There are four components in this system: 1) parametric graphic design of patterns, 2) sensing interface printout through an inkjet printer (Brother DCP-J140w) with conductive silver ink, 3) a custom-made hardware interface for sensing communication with a computer, and 4) software (MAX/MSP) for computer music generation.

\section{Parametric Pattern Design}

The pattern is parametrically-designed in Grasshopper (a plug-in of Rhino). We defined surface boundaries and force vectors manually before using Grasshopper's Spatial Deform function to apply manifold for the visualization of a smooth field. This surface is then sliced using Level Set with a constant height $(\mathrm{Z})$, receiving the contour lines as shown in (Fig 2). A few manual steps are further required to connect the traces and define additional color prints. Once the regions and patterns were designed, we assigned regions of interest as functional inputs (buttons, sliders...etc). To mark the regions visually, we add another layer of color printing to add graphic details and boundaries. We also cut out several regions with a laser cutter to preserve and reveal the original instrument design. The entire surface is a controller - even 'dead traces', such as decorative islands, can be bridged by one's fingers for performance effect.

\section{Rapid Prototyping with Conductive Inkjet Printing}

The process we explored is printing conductive traces with silver ink and an off-the-shelf inkjet printer (Figure 3(a)). We adapted conductive silver ink from Mitsubishi Paper Mill and print conductive traces on photo paper or PET films. With this fabrication method, any iteration of the sensing surface only takes less than 5 minutes to construct.

\section{Hardware Interface for Sensing and Communication}

There are many ways to detect the varying signal on the conductive patterns such as resistive and capacitive sensing. In our implementation, we adopted capacitive sensing and add expressiveness to the music control by including sliding, pressing, and proximity to the performance. The

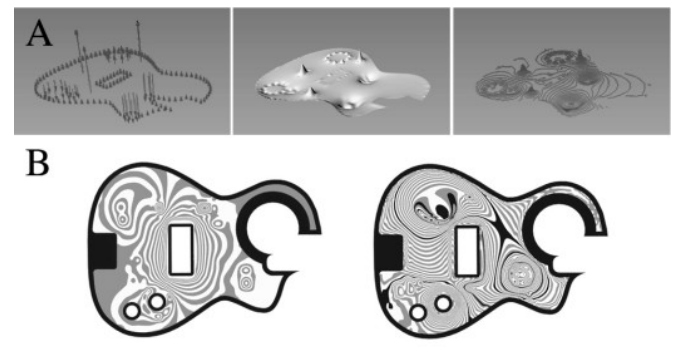

Figure 2. Design process: (A) from vectors to contours, (B) two examples of received patterns.
A
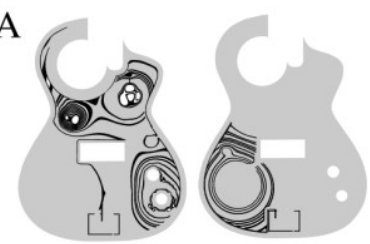

B

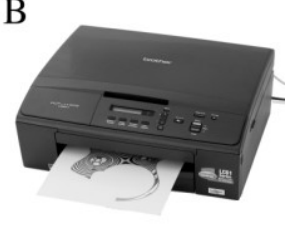

Figure 3. (A) Two examples of tracing continuity in sub-patterns, (B) the printing process.

PCB is attached to the flexible sensor sheet through signal pads at the bottom of the board with the 3M $9703 \mathrm{z}$-axis conductive tape.

\section{Signal Mapping}

The last component is the computer music software, which generates the sonic output of our sensing surfaces. We use Max/MSP as our experiment platform and implemented effects that are mapped to the patterns and buttons that control inputs such as echoing, overdrive, delay, and distortion.

\section{DISCUSSION}

Combining sensing-enabled objects and flexible conductive printing, our work serves as a platform for prototyping aesthetic graphic sensing input with high accuracy and reproducibility. Our platform explores the unification of compelling graphics and expressive input devices - a promising way of creating a new form of art that intimately combines graphics, interaction, and music. More information can be found on our website: http://resenv.media.mit.edu/zebra.html

\section{REFERENCES}

1. http://baranoff-rossine.com/optophonic-piano/

2. N.-W. Gong, N. Zhao and J. A. Paradiso," A Customizable Sensate Surface for Music Control," (NIME 12), pp. 417-420.

3. A. Freed, J. Rowland, "Collocated Surface Sound Interaction," in CHI 2013, Interactivity Demo Session 